\title{
Photoinduced Hydrocarboxylation via Thiol-Catalyzed Delivery of For- mate Across Alkenes
}

\author{
Sara N. Alektiar and Zachary K. Wickens* \\ Department of Chemistry, University of Wisconsin-Madison, Madison, Wisconsin 53706, United States
} ABSTRACT: Herein we disclose a new photochemical process to prepare carboxylic acids from formate salts and alkenes.
This redox-neutral hydrocarboxylation proceeds in high yields across diverse functionalized alkene substrates with excellent
regioselectivity. This operationally simple procedure can be readily scaled with low photocatalyst loading (0.01\% photocata-
lyst) without the need for a flow reactor or any precautions to exclude air or moisture. Furthermore, this new reaction can
leverage commercially available formate carbon isotologues to enable the direct synthesis of isotopically labeled carboxylic
acids. Mechanistic studies support the working model involving a thiol-catalyzed radical chain process wherein the atoms
from formate are delivered across the alkene substrate via $\mathrm{CO}_{2}{ }^{\circ-}$ as a key reactive intermediate.

The selective and efficient transformation of alkenes into polar functional groups is a fundamental synthetic strategy. Tremendous progress has been made in alkene functionalization;1-11 however, many seemingly simple transformations remain challenging to accomplish. Our group has a growing interest in advancing new alkene functionalization strategies designed to leverage appealing chemical building blocks rather than high energy reagents. ${ }^{12}$ Following this line of inquiry, we questioned whether synthetically valuable carboxylic acids ${ }^{13-17}$ could be prepared by delivering formate salts across alkenes (Figure 1, top). In principle, this thermodynamically favorable ${ }^{18-21}$ transformation could occur with perfect atom ${ }^{22}$ and redox ${ }^{23}$ economy. However, while formate is a common reductant in transition metal catalysis, ${ }^{24-26}$ it is rarely used as a $\mathrm{C} 1$ source despite the practical appeal of such an approach relative to gaseous alternatives. Notably, Shi and coworkers leveraged in situ generated formate ahydrides as CO-surrogates for hydrocarboxylation using palladium-catalysis at elevated temperature. ${ }^{27-32}$ We envisioned that a mild approach to directly add formate salts across alkenes would constitute an attractive alternative to this strategy as well as established CO-based hydrocarboxylation processes ${ }^{33}$ and emerging methods that rely on reductive activation of $\mathrm{CO}_{2} \cdot{ }^{34-50}$

Our reaction design was guided by the recognition that formate is formally comprised of $\mathrm{CO}_{2}{ }^{--}$and a hydrogen atom. A strategy to elicit this reactivity from formate could tap into recently developed hydrocarboxylation manifolds that proceed via SET reduction of $\mathrm{CO}_{2}$ (Figure 1, middle). Unfortunately, reductive approaches to access $\mathrm{CO}_{2}{ }^{\bullet-}$ require deeply reducing conditions due to the thermodynamic stability of $\mathrm{CO}_{2}\left(E_{1 / 2}\left(\mathrm{CO}_{2} / \mathrm{CO}_{2}{ }^{-}\right)=-2.2 \mathrm{~V}\right.$ vs SCE $) .{ }^{51}$ To access this requisite driving force for $\mathrm{CO}_{2}$ reduction, prior efforts required $<300 \mathrm{~nm}$ UV light, ${ }^{46,47}$ visible light with stoichiometric thiolate-promoters, ${ }^{48}$ or deeply reducing electrodes. ${ }^{49,50}$ Furthermore, the photochemical approaches require external hydrogen atom sources while high electrode overpotentials for $\mathrm{CO}_{2}$ reduction ${ }^{51,52}$ erode functional group tolerance in electrochemical strategies.

In contrast to $\mathrm{CO}_{2}$-reduction strategies, formate enters the reaction in the appropriate oxidation state for alkene hydrocarboxylation without need of a sacrificial electron donor. We suspected that redox-neutral hydrocarboxylation by delivery of formate across alkenes would not only improve atom economy relative to net-reductive strategies,

\section{Target Transformation: Redox-neutral addition of formate across an alkene}

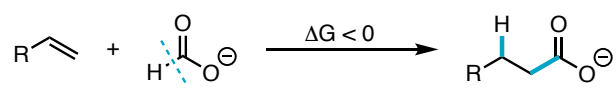

perfect atom economy = inexpensive $\mathrm{C} 1$ source - favorable thermochemistry

Our framework for formate hydrocarboxylation

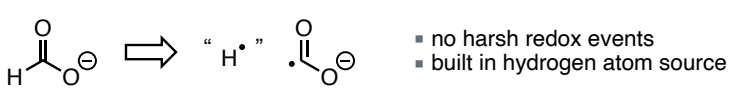

formal formate homolysis reveals necessary fragments for hydrocarboxylation

Established hydrocarboxylation via $\mathrm{CO}^{--}$

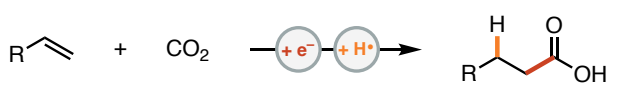

deeply reducing conditions representative $\mathrm{H}$-atom sources

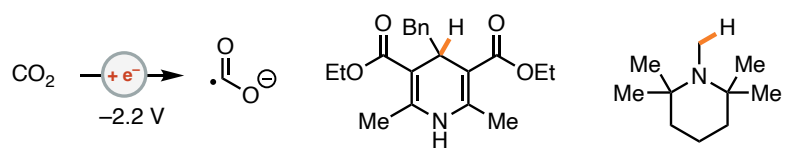

This Work: Photoredox promoted thiol catalyzed hydrocarboxylation

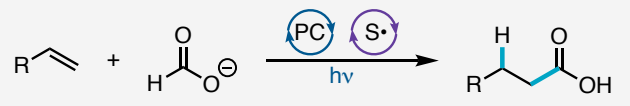

operationally simple = broad scope - low catalyst loadings = scaleable in batch

Figure 1. Redox-neutral alkene hydrocarboxylation overview. 
but would also provide an appealing chemoselectivity profile by circumventing the need for strong reductants..$^{53}$ Our group ${ }^{54}$ and others ${ }^{55,56}$ recently introduced a collection of photoredox ${ }^{57-65}$ strategies to generate $\mathrm{CO}_{2}{ }^{*-}$ in situ via cleavage of the formate $\mathrm{C}\left(\mathrm{sp}^{2}\right)-\mathrm{H}$ bond. In these prior studies, the nascent $\mathrm{CO}_{2}{ }^{-}$was primarily employed as an SET reductant. ${ }^{66}$ We hypothesized that our catalytic system could be repurposed as a general and functional group tolerant strategy to access $\mathrm{CO}_{2}{ }^{--}$for $\mathrm{C}-\mathrm{C}$ bond-forming reactions. Overall, this would introduce a mechanistically distinct approach to promote hydrocarboxylation reactions via a mild oxidation event $\left(E_{\text {ox }}\left(\mathrm{CHO}_{2}^{-}\right)=+1.25 \mathrm{~V}\right.$ vs SCE$)$ or hydrogen atom abstraction $(\mathrm{BDE}=86 \mathrm{kcal} / \mathrm{mol})^{20}$ instead of the difficult SET reduction of $\mathrm{CO}_{2}$. Herein, we report a redox-neutral approach to hydrocarboxylation via addition of formate across alkene substrates (Figure 1, bottom).

We selected styrene as a model alkene substrate as diverse analogs are commercially available and 3-aryl propionic acids are well-represented in bioactive molecules. ${ }^{14,15}$ Of note, the anticipated linear selectivity will complement $\mathrm{CO}_{2}$-based transition-metal-catalyzed processes that furnish branched products from alkenylarenes $35,37,38,40,42,44,45$ with one notable recent exception from König and co-workers. ${ }^{40}$ Accordingly, we evaluated our previously developed conditions for $\mathrm{CO}_{2}{ }^{--}$generation from formate ${ }^{54}$ in the presence of styrene. These conditions fully converted styrene in 20 hours and provided a $25 \%$ yield of the linear carboxylate, 3, along with $37 \%$ ethylbenzene. Reaction optimization-including adjusting the irradiation wavelengths away from those that excite reduced 4DPAIPN ${ }^{67}$-resulted in improved conditions that furnish nearly quantitative yield of $\mathbf{3}$ without observable ethylbenzene. Furthermore, these reaction conditions provided complete conversion in two hours with low loadings of photoredox and thiol catalysts (Table 1, entry 1). Control experiments confirmed that no conversion of styrene was observed in the absence of the photoredox catalyst (entry 2). ${ }^{68}$

The structure of the thiol hydrogen atom transfer (HAT) catalyst was identified as a key parameter. Omission of the thiol from the reaction resulted in diminished rate and, consequently, reduced chemical yield (entry 3 ). The alkyl thiol we employed in related, formate-based hydroarylation processes, ${ }^{54} \mathrm{CySH}$, was similarly ineffective (entry 4). While several thiolphenols and electron-deficient thiols performed comparably to T1 (see Table S1), when T1 was substituted for an electron-rich analog, $\mathbf{T} 2$, the yield was substantially diminished, reverting to nearly that of the reaction performed without thiol (entry 5). Overall, these data cannot be rationalized using thermodynamic parameters such as BDEs ${ }^{69,70}$ but are fully consistent with substantial polar effects on the HAT transition structures. ${ }^{71}$

The formate counterion also had an impact on the reaction. Substitution of potassium for sodium slows the reaction and results in lower yield (entry 6). Replacement of potassium with cesium delivers a similar yield and modestly accelerates the rate (entry 7, see Table $\mathrm{S6}$ for details regarding rate changes). We attribute this effect to differential solubility of the formate salts in DMSO. Potassium formate was selected for further study as it furnishes nearly quantitative product in only two hours and is inexpensive. ${ }^{72}$

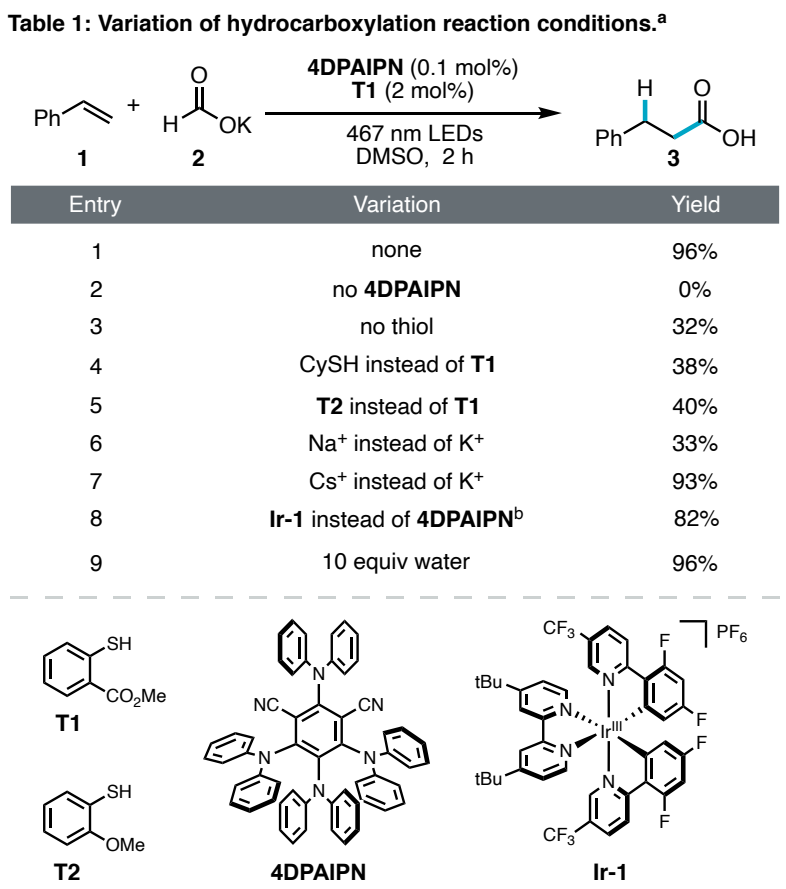

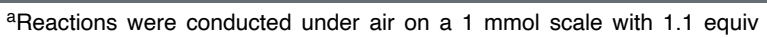
$\mathrm{KCHO}_{2}$. Yield of 3 determined by ${ }^{1} \mathrm{H}$ NMR analysis. See the Supporting Information (SI) for details. ${ }^{\mathrm{b}}$ The reaction was run for $24 \mathrm{~h}$ with $1 \mathrm{~mol} \%$ Ir-1.

The photoredox catalyst identified (4DPAIPN) was particularly effective; however, a variety of other photocatalysts promote the reaction. For example, iridium-based photocatalysts could be used in place of 4DPAIPN, albeit with extended reaction times (entry 8). ${ }^{73}$ We found that the generation of these carboxylic acid products is robust; no precautions to exclude air or moisture are necessary and the process tolerates the deliberate addition of water (entry 9).

We next examined the scope of this new alkene hydrocarboxylation reaction. We found that these simple conditions promote the delivery of formate across a wide range of alkenylarene substrates with exquisite functional group tolerance (Table 2). Diverse electron-donating and electron-withdrawing substituents could be introduced on the arene (3-8) without a substantial impact on reaction efficiency. Since the reaction conditions are only mildly basic, protic substrates were well-tolerated. Alkenylarenes bearing carbamates (5), carboxylic acids (7 and 14), ${ }^{74}$ and unprotected alcohols ( 9 and 13) each underwent hydrocarboxylation in high yield. Furthermore, a substrate containing a synthetically versatile but Lewis acidic boronic acid pinacol ester (8) was efficiently converted into the linear carboxylic acid. This redox-neutral process also tolerates reductively sensitive functional groups, such as aryl chlorides (6). This substrate was of particular interest because we have previously engaged aryl chlorides in reductive radical coupling reactions under similar formate-based reaction conditions. ${ }^{75}$ Hydrocarboxylation of 2-vinylpyridne (10) illustrated the suitability of vinyl heterocycles as substrates. The reaction was insensitive to additional alkene substituents; $\alpha$ - and $\beta$-methyl styrenes were each converted to carboxylic acids (11 and 12). Additionally, functionalized alkyl groups such as unprotected alcohols or acids in the $\beta$ - 
Table 2: Scope of alkenylarene substrates. ${ }^{a}$

$$
\text { (2) }
$$
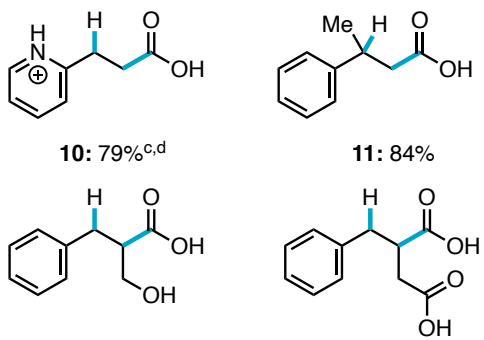

aReactions were conducted under air on a $1 \mathrm{mmol}$ scale with 1.1 equiv $\mathrm{KCHO}_{2}$ and yields are of purified product unless otherwise noted. See the $\mathrm{SI}$ for further details. ${ }^{b} 10$ equiv $\mathrm{CsCHO}_{2}$. ${ }^{c}$ Yield determined via ${ }^{1} \mathrm{H}$ NMR. ${ }^{\mathrm{d}}$ Reaction was run for $5 \mathrm{~h}$ in DMSO-d6 and measured as yield of trifluoroacetate salt.

position did not disrupt the hydrocarboxylation process (13 and 14).

We found that the reaction was not limited to alkenylarene substrates; electron-deficient alkenes successfully underwent hydrocarboxylation to furnish succinate derivatives (Table 3). Hydrocarboxylation of these substrates was unperturbed by substitution at either the $\alpha$ - or $\beta$-position (15-17), providing access to differentially substituted 1,4-dicarbonyl compounds. Hindered $\beta, \beta$-disubstituted substrates converted at diminished rate under the standard conditions; however, substitution of potassium formate for an excess of more soluble cesium formate accelerated the rate and delivered product (18) in high yield in two hours. Further investigation of this sterically-congested substrate class illustrated that this new reaction provides efficient access to heterocyclic building blocks bearing $\alpha$ quaternary carboxylic acids (19-21). Since aliphatic alkenes did not undergo hydrocarboxylation, a substrate bearing both an unactivated and activated alkene (22) underwent chemoselective hydrocarboxylation at the electron-deficient alkene to provide 23 .
Table 3: Scope of electron-deficient alkene substrates. ${ }^{\text {a }}$

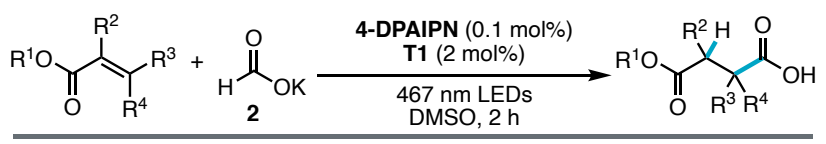

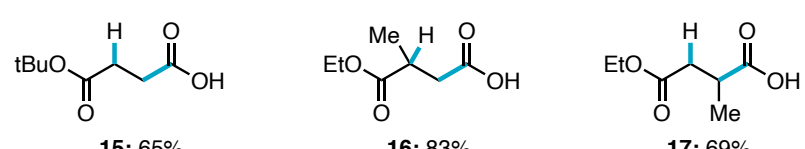<smiles>CCOC(=O)C=CC(=O)C1(C(=O)O)CCOCC1</smiles>

$$
\begin{gathered}
19: 64 \%^{\mathrm{b}} \\
5: 1(\beta: \alpha)^{\mathrm{c}}
\end{gathered}
$$

aReactions were conducted under air on a $1 \mathrm{mmol}$ scale with 1.1 equiv $\mathrm{KCHO}_{2}$ and $0.1 \mathrm{~mol} \%$ 4-DPAIPN and yields are of purified product unless otherwise noted. See the $\mathrm{SI}$ for further details. ${ }^{b} 10$ equiv $\mathrm{CsCHO}_{2}$ and $1 \mathrm{~mol} \%$ 4-DPAIPN. CIsolated as a mixture of $\beta$ - and $\alpha$-carboxylic acid products. ${ }^{\mathrm{d}}$ Yield determined via ${ }^{1} \mathrm{H}$ NMR.

Given that scaling photochemical reactions can be technically challenging, ${ }^{76}$ we evaluated the viability of performing this process on preparative scale. Using a simple batch setup with no precautions to exclude air or moisture, the carboxylate salt 24 was synthesized in $79 \%$ yield $7.4 \mathrm{~g}$, $39.5 \mathrm{mmol}$ ) in under nine hours using only $0.01 \mathrm{~mol} \%$ of the photocatalyst, 4DPAIPN (Scheme 1). This preparative scale reaction is not only technically simple to execute but the carboxylate salt can be purified from the reaction mixture by crystallization with neither chromatography nor extensive aqueous washes to remove non-volatile DMSO. Overall, these results illustrate the immediate practical utility of this new formate-based hydrocarboxylation reaction.

Scheme 1. Multigram scale hydrocarboxylation. ${ }^{a}$

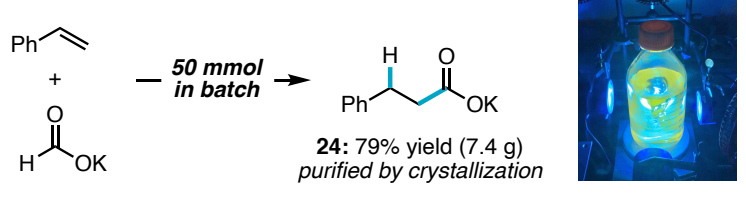

aThe reaction was conducted under air on a $50 \mathrm{mmol}$ scale and run for $8.5 \mathrm{~h}$ with 1.1 equiv $\mathrm{KCHO}_{2}, 5 \mathrm{~mol} \% \mathrm{T1}$, and $0.01 \mathrm{~mol} \%$ 4DPAIPN. See the SI for further details.

Based on our working mechanistic model, we anticipate that the formate salt is incorporated as the carboxylic acid in the final product. This opens up an appealing avenue to prepare isotopically labeled molecules because both ${ }^{13} \mathrm{C}$ and ${ }^{14} \mathrm{C}$-labeled sodium formate salts are commercially available. We first adjusted the reaction conditions to employ limiting sodium formate as this would be particularly attractive for ${ }^{14} \mathrm{C}$-radiolabeling applications. ${ }^{77}$ Under these modified conditions, we found three distinct bioactive molecules (25-27) could be produced with near perfect ${ }^{13} \mathrm{C}$-incorporation (Table 4). This offers a simple but effective complement to recently developed carboxylic acid isotopic exchange reactions. ${ }^{78-86}$ These equilibrium methods cannot 
simultaneously deliver high specific activity and radiochemical yield whereas this hydrocarboxylation process offers both. Finally, each of these bioactive carboxylic acids can also be synthesized efficiently under our standard limiting-alkene conditions in high yields (see Figure S9).

Table 4. ${ }^{13} \mathrm{C}$-labeling of bioactive molecule via hydrocarboxylation. ${ }^{\mathrm{a}}$
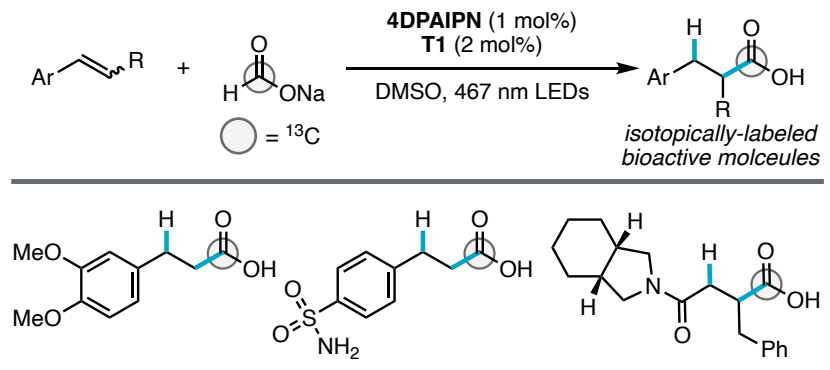

NSC-76043 (25) $51 \%$ yield $\left[{ }^{13} \mathrm{C}:>99 \%\right] \quad 71 \%$ yield $\left[{ }^{13} \mathrm{C}:>99 \%\right]$

$( \pm)$-Mitiglinide (27)

a Reactions were conducted under air and run for $8 \mathrm{~h}$ with $0.4 \mathrm{mmol} \mathrm{Na}{ }^{13} \mathrm{CHO}_{2}$ and $0.6 \mathrm{mmol}$ styrene and yields were determined relative to $\mathrm{Na}^{13} \mathrm{CHO}_{2}$ via ${ }^{1} \mathrm{H}$ NMR and ${ }^{13} \mathrm{C}$ incorporation was determined by mass spectrometry. See the $\mathrm{SI}$ for further details.

We next questioned whether the hydrogen atom incorporated in the final product is derived from formate. To this end, we subjected D-formate to our reaction conditions and observed high but incomplete deuteration (Table 5, entry 1 ). This is consistent with formate acting both as a C1 and hydrogen atom source but also suggests a secondary process to account for the incomplete deuterium incorporation. We questioned whether a parallel electron-transfer-protontransfer (ETPT) pathway may also occur. In this process, the benzylic radical would be reduced $\left(E_{\mathrm{red}}=-1.4 \mathrm{~V} \text { vs SCE}\right)^{87}$ and subsequently protonated, by solvent or adventitious water.88 However, running the reaction in DMSO-d6 resulted in no measurable deuterium incorporation (entry 2). Given that both DMSO and water would spontaneously quench a benzylic anion and DMSO is present in vast excess, these data indicate that ETPT is not responsible for incomplete transfer of the deuterium label from formate to the carboxylic acid product. Given our working model that the thiol catalyzes the reaction by shuttling hydrogen atoms, we suspected that proton exchange between the thiol co-catalyst and adventitious water could explain these data. Addition of $\mathrm{D}_{2} \mathrm{O}$ to otherwise standard conditions resulted in substantial deuterium incorporation (entry 3). Conversely, when a D-formate reaction was conducted in the presence of $\mathrm{H}_{2} \mathrm{O}$, deuterium incorporation was not observed (entry 4). Taken together, these data are consistent with our working model wherein the thiol catalyzes HAT from formate to transient $\mathrm{C}\left(\mathrm{sp}^{3}\right)$-radical intermediates. ${ }^{89}$
Table 5. Deuterium labeling studies. ${ }^{a}$

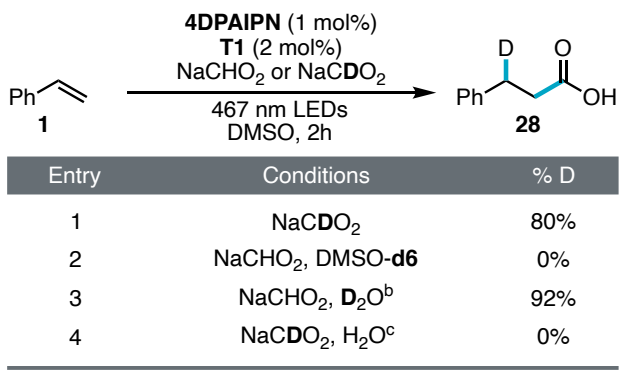

aReactions were conducted under $\mathrm{N}_{2}$ on a $0.1 \mathrm{mmol}$ scale with 1.1 equiv $\mathrm{NaCO}_{2} \mathrm{H}$ or $\mathrm{NaCO}_{2} \mathrm{D}$. See the SI for further details. ${ }^{b} 11$ equiv $\mathrm{D}_{2} \mathrm{O}$. ${ }^{c} 11$ equiv $\mathrm{H}_{2} \mathrm{O}$.

Based on these results, we propose a working mechanistic model based on a thiol-catalyzed radical chain process (scheme 2). ${ }^{90}$ Thiyl radicals generated in situ could abstract a hydrogen atom from formate to generate $\mathrm{CO}_{2}{ }^{\bullet-}$. This radical anion intermediate, in turn, reacts with the alkene substrate to furnish a new $\mathrm{C}-\mathrm{C} \sigma$-bond and a $\mathrm{C}\left(\mathrm{sp}^{3}\right)$ radical. This radical intermediate is quenched by HAT from the thiol, regenerating the thiyl radical. We envisioned two plausible mechanisms for initiation of this chain process. First, SET oxidation of formate in DMSO is known to result in a second order decomposition to formic acid and $\mathrm{CO}_{2}{ }^{--}$(initiation A). ${ }^{91}$ Second, SET oxidation of the thiol followed by a proton transfer could directly generate the key thiyl radical intermediate (initiation B). Stern-Volmer analysis indicated that both a soluble formate salt (tetrabutylammonium formate) and the thiol catalyst, T1, quenched the excited state of the photoredox catalyst 4DPAIPN. However, the rate of photocatalyst quenching by $\mathbf{T 1}$ is approximately an order of magnitude faster than formate and potassium formate is sparingly soluble in DMSO. ${ }^{92}$ Accordingly, we favor SET oxidation of the thiol catalyst (initiation B) as the primary initiation mechanism but suspect both occur in parallel under standard conditions.

Scheme 2. Plausible thiol-catalyzed radical chain mechanism.

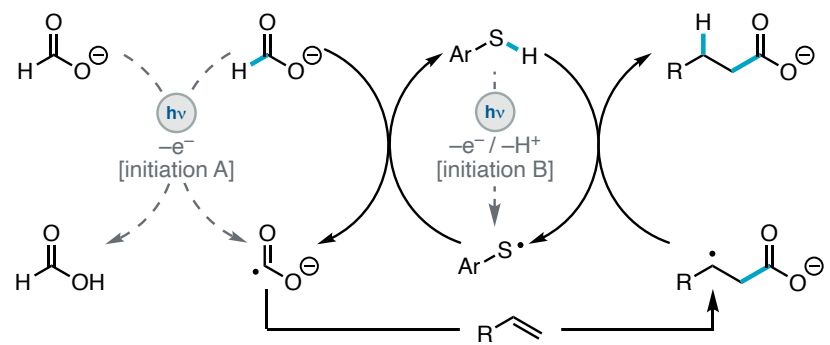

Overall, we have introduced a new strategy to access linear carboxylic acids from formate and activated alkenes by exploiting a photoinitiated, thiol-catalyzed chain reaction. This redox-neutral process is conducted under mild conditions without stoichiometric redox agents and, as a result, a wide variety of functional groups are tolerated by the procedure. The conditions are operationally simple; no precautions to exclude air or moisture are necessary. This transformation is also readily translated to preparative scale (50 mmol) using a straightforward batch reaction set up. This hydrocarboxylation method also provides a facile approach to leverage commercially available isotopically labeled formate salts to prepare labeled bioactive carboxylate 
products. Additionally, mechanistic investigations revealed that formate acts as both the $\mathrm{C} 1$ and hydrogen atom source. This study illustrates the potential benefits of a redox-neutral approach to alkene hydrocarboxylation and, more broadly, provides a roadmap to unlock formate as a general $\mathrm{CO}_{2}{ }^{--}$surrogate for $\mathrm{C}-\mathrm{C}$ bond forming reactions.

\section{ASSOCIATED CONTENT}

\section{Supporting Information}

The Supporting Information is available free of charge on the ACS Publications website.

Experimental procedures, characterization data, and spectra (PDF)

\section{AUTHOR INFORMATION}

\section{Corresponding Author}

* Zachary K. Wickens - Department of Chemistry, University of Wisconsin-Madison, Madison, Wisconsin 53706, United States; orcid.org/0000-0002-5733-5288; Email: wickens@wisc.edu

\section{Author Contributions}

Sara N. Alektiar - Department of Chemistry, University of Wisconsin-Madison, Madison, Wisconsin 53706, United States; orcid.org/0000-0003-4283-468X

\section{Notes}

The authors declare no competing financial interest.

\section{ACKNOWLEDGMENT}

We thank Prof. Mark Levin, Prof. Daniel Weix, and Prof. Tehshik Yoon for helpful suggestions. We thank the Stahl, Weix, Yoon, and Schomaker groups for sharing their chemical inventory. We thank Dr. Wesley Swords (Yoon group) for assistance with Stern-Volmer quenching experiments. This work was financially supported by the Office of the Vice Chancellor for Research and Graduate Education at the University of WisconsinMadison with funding from the Wisconsin Alumni Research Foundation. Acknowledgement is made to the Donors of the American Chemical Society Petroleum Research Fund for partial funding of this research (60677-DNI1). Spectroscopic instrumentation was supported by a generous gift from Paul J. and Margaret M. Bender, NSF (CHE-1048642), and NIH (S100D012245 and 1S100D020022-1).

\section{REFERENCES}

(1) While not an exhaustive list, for representative reviews of recent advances in alkene functionalization, see Refs 2-11.

(2) Beller, M.; Seayad, J.; Tillack, A.; Jiao, H. Catalytic Markovnikov and Anti-Markovnikov Functionalization of Alkenes and Alkynes: Recent Developments and Trends. Angew. Chem. Int. Ed. 2004, 43 (26), 3368-3398.

(3) Hintermann, L. Recent Developments in Metal-Catalyzed Additions of Oxygen Nucleophiles to Alkenes and Alkynes. In $C$ - $X$ Bond Formation; Vigalok, A., Ed.; Topics in Organometallic Chemistry; Springer: Berlin, Heidelberg, 2010; pp 123-155.

(4) Margrey, K. A.; Nicewicz, D. A. A General Approach to Catalytic Alkene Anti-Markovnikov Hydrofunctionalization Reactions via Acridinium Photoredox Catalysis. Acc. Chem. Res. 2016, 49 (9), 1997-2006.
(5) Liu, R. Y.; Buchwald, S. L. CuH-Catalyzed Olefin Functionalization: From Hydroamination to Carbonyl Addition. Acc. Chem. Res. 2020, 53 (6), 1229-1243.

(6) Shevick, S. L.; Wilson, C. V.; Kotesova, S.; Kim, D.; Holland, P. L.; Shenvi, R. A. Catalytic Hydrogen Atom Transfer to Alkenes: A Roadmap for Metal Hydrides and Radicals. Chem. Sci. 2020, 11 (46), 12401-12422.

(7) Cristina Silva Costa, D. Additions to Non-Activated Alkenes: Recent Advances. Arab. J. Chem. 2020, 13 (1), 799-834.

(8) Siu, J. C.; Fu, N.; Lin, S. Catalyzing Electrosynthesis: A Homogeneous Electrocatalytic Approach to Reaction Discovery. Acc. Chem. Res. 2020, 53 (3), 547-560.

(9) Davison, R. T.; Kuker, E. L.; Dong, V. M. Teaching Aldehydes New Tricks Using Rhodium- and Cobalt-Hydride Catalysis. Acc. Chem. Res. 2021, 54 (5), 1236-1250.

(10) Streiff, S.; Jérôme, F. Hydroamination of Non-Activated Alkenes with Ammonia: A Holy Grail in Catalysis. Chem. Soc. Rev. 2021, 50 (3), 1512-1521.

(11) Yao, H.; Hu, W.; Zhang, W. Difunctionalization of Alkenes and Alkynes via Intermolecular Radical and Nucleophilic Additions. Molecules 2021, 26 (1), 105.

(12) Holst, D. E.; Wang, D. J.; Kim, M. J.; Guzei, I. A.; Wickens, Z. K. Aziridines by Coupling Amines and Alkenes via an Electro-Generated Dication. Nature 2021, Accelerated article preview https://doi.org/10.1038/s41586-021-03717-7.

(13) Carboxylic acids are widely represented in natural products, pharmaceuticals, and commodity chemicals. For examples, see: Refs 14-17.

(14) Bhutani, P.; Joshi, G.; Raja, N.; Bachhav, N.; Rajanna, P. K.; Bhutani, H.; Paul, A. T.; Kumar, R. U.S. FDA Approved Drugs from 2015-June 2020: A Perspective. J. Med. Chem. 2021, 64 (5), 23392381.

(15) Lamberth, C.; Dinges, J. Bioactive Carboxylic Compound Classes: Pharmaceuticals and Agrochemicals | Wiley http://www.wiley.com/en-us/Bioactive+Carboxylic+Compound+Classes $\% 3 \mathrm{~A}+$ Pharmaceuticals+and+Agrochemicals-p9783527339471 (accessed 2021-07-14).

(16) Varenikov, A.; Shapiro, E.; Gandelman, M. Decarboxylative Halogenation of Organic Compounds. Chem. Rev. 2021, 121 (1), 412-484.

(17) Stempfle, F.; Ortmann, P.; Mecking, S. Long-Chain Aliphatic Polymers To Bridge the Gap between Semicrystalline Polyolefins and Traditional Polycondensates. Chem. Rev. 2016, 116 (7), 45974641.

(18) A simple accounting of BDEs suggests this reaction should be exothermic. For $\mathrm{C}-\mathrm{CO} 2 \mathrm{H}$ bond strength, see Ref 19; for $\mathrm{H}-\mathrm{CO} 2-$ bond strength, see Ref 20 ; for $\mathrm{C}=\mathrm{C}$ and $\mathrm{C}-\mathrm{C}$ bond strengths, see Ref 21.

(19) Namysl, S.; Pelucchi, M.; Herbinet, O.; Frassoldati, A.; Faravelli, T.; Battin-Leclerc, F. A First Evaluation of Butanoic and Pentanoic Acid Oxidation Kinetics. Chem. Eng. J. 2019, 373, 973984.

(20) Grills, D. C.; Lymar, S. V. Radiolytic Formation of the Carbon Dioxide Radical Anion in Acetonitrile Revealed by Transient IR Spectroscopy. Phys. Chem. Chem. Phys. 2018, 20 (15), 1001110017.

(21) Luo, Y.-R. Comprehensive Handbook of Chemical Bond Energies; CRC Press: Boca Raton, 2007.

(22) Trost, B. M. On Inventing Reactions for Atom Economy. Acc. Chem. Res. 2002, 35 (9), 695-705.

(23) Burns, N. Z.; Baran, P. S.; Hoffmann, R. W. Redox Economy in Organic Synthesis. Angew. Chem. Int. Ed. 2009, 48 (16), 28542867.

(24) Ram, S.; Ehrenkaufer, R. E. Ammonium Formate in Organic Synthesis: A Versatile Agent in Catalytic Hydrogen Transfer Reductions. Synth. Ger. 1988, 1988 (2), 91-95.

(25) Tsuji, J.; Mandai, T. Palladium-Catalyzed Hydrogenolysis of Allylic and Propargylic Compounds with Various Hydrides. Synthesis 1996, 1996 (01), 1-24. 
(26) Modak, A.; Maiti, D. Metal Catalyzed Defunctionalization Reactions. Org. Biomol. Chem. 2016, 14 (1), 21-35.

(27) Wang, Y.; Ren, W.; Li, J.; Wang, H.; Shi, Y. Facile PalladiumCatalyzed Hydrocarboxylation of Olefins without External CO Gas. Org. Lett. 2014, 16 (22), 5960-5963.

(28) Dai, J.; Ren, W.; Wang, H.; Shi, Y. A Facile Approach to $\beta$ Amino Acid Derivatives via Palladium-Catalyzed Hydrocarboxylation of Enimides with Formic Acid. Org. Biomol. Chem. 2015, 13 (31), 8429-8432.

(29) Dai, J.; Ren, W.; Chang, W.; Zhang, P.; Shi, Y. An Effective Route to $\beta 2$-Amino Acid Derivatives via Pd-Catalyzed Regioselective Hydrocarboxylation of 1,2-Disubstituted Enimides. Org. Chem. Front. 2017, 4 (2), 297-302.

(30) Dai, J.; Ren, W.; Li, J.; Shi, Y. An Effective Approach to ArylSubstituted Propanoic Acids via the Pd-Catalyzed Hydrocarboxylation of Stilbenes. Org. Chem. Front. 2018, 5 (4), 561-565.

(31) Liu, W.; Ren, W.; Li, J.; Shi, Y.; Chang, W.; Shi, Y. A LigandDirected Catalytic Regioselective Hydrocarboxylation of Aryl Olefins with Pd and Formic Acid. Org. Lett. 2017, 19 (7), 1748-1751.

(32) Ren, W.; Chu, J.; Sun, F.; Shi, Y. Pd-Catalyzed Highly Chemoand Regioselective Hydrocarboxylation of Terminal Alkyl Olefins with Formic Acid. Org. Lett. 2019, 21 (15), 5967-5970.

(33) Kalck, P.; Urrutigoïty, M.; Dechy-Cabaret, O. Hydroxy- and Alkoxycarbonylations of Alkenes and Alkynes. In Catalytic Carbonylation Reactions; Beller, M., Ed.; Topics in Organometallic Chemistry; Springer: Berlin, Heidelberg, 2006; pp 97-123.

(34) For examples of transition-metal-catalyzed processes that engage $\mathrm{CO} 2$ in hydrocarboxylation, see Refs 35-45. For examples of SET reduction of CO2, see Refs 46-50.

(35) Gaydou, M.; Moragas, T.; Juliá-Hernández, F.; Martin, R. Site-Selective Catalytic Carboxylation of Unsaturated Hydrocarbons with CO2 and Water. J. Am. Chem. Soc. 2017, 139 (35), 1216112164.

(36) Wu, L.; Liu, Q.; Fleischer, I.; Jackstell, R.; Beller, M. Ruthenium-Catalysed Alkoxycarbonylation of Alkenes with Carbon Dioxide. Nat. Commun. 2014, 5 (1), 3091.

(37) Kawashima, S.; Aikawa, K.; Mikami, K. Rhodium-Catalyzed Hydrocarboxylation of Olefins with Carbon Dioxide. Eur. J. Org. Chem. 2016, 2016 (19), 3166-3170. https://doi.org/10.1002/ejoc.201600338.

(38) Williams, C. M.; Johnson, J. B.; Rovis, T. Nickel-Catalyzed Reductive Carboxylation of Styrenes Using C02. J. Am. Chem. Soc. 2008, 130 (45), 14936-14937.

(39) Wang, X.; Nakajima, M.; Martin, R. Ni-Catalyzed Regioselective Hydrocarboxylation of Alkynes with $\mathrm{CO} 2$ by Using Simple Alcohols as Proton Sources. J. Am. Chem. Soc. 2015, 137 (28), 89248927.

(40) Meng, Q.-Y.; Wang, S.; Huff, G. S.; König, B. Ligand-Controlled Regioselective Hydrocarboxylation of Styrenes with $\mathrm{CO}_{2}$ by Combining Visible Light and Nickel Catalysis. J. Am. Chem. Soc. 2018, 140 (9), 3198-3201.

(41) Tanaka, S.; Tanaka, Y.; Chiba, M.; Hattori, T. Lewis Acid-Mediated $\beta$-Selective Hydrocarboxylation of $\alpha, \alpha$-Diaryl- and $\alpha$-Arylalkenes with R3SiH and CO2. Tetrahedron Lett. 2015, 56 (24), 38303834 .

(42) Greenhalgh, M. D.; Thomas, S. P. Iron-Catalyzed, Highly Regioselective Synthesis of $\alpha$-Aryl Carboxylic Acids from Styrene Derivatives and C02. J. Am. Chem. Soc. 2012, 134 (29), 11900-11903. (43) Bringmann, J.; Dinjus, E. Electrochemical Synthesis of Carboxylic Acids from Alkenes Using Various Nickel-Organic Mediators: $\mathrm{CO} 2$ as C1-Synthon. Appl. Organomet. Chem. 2001, 15 (2), 135-140.

(44) Shao, P.; Wang, S.; Chen, C.; Xi, C. Cp2TiCl2-Catalyzed Regioselective Hydrocarboxylation of Alkenes with CO2. Org. Lett. 2016, 18 (9), 2050-2053.

(45) Murata, K.; Numasawa, N.; Shimomaki, K.; Takaya, J.; Iwasawa, N. Construction of a Visible Light-Driven Hydrocarboxylation Cycle of Alkenes by the Combined Use of Rh(I) and Photoredox Catalysts. Chem. Commun. 2017, 53 (21), 3098-3101.
(46) Seo, H.; Liu, A.; Jamison, T. F. Direct $\beta$-Selective Hydrocarboxylation of Styrenes with CO2 Enabled by Continuous Flow Photoredox Catalysis. J. Am. Chem. Soc. 2017, 139 (40), 13969-13972. (47) Kang, G.; Romo, D. Photocatalyzed, $\beta$-Selective Hydrocarboxylation of $\alpha, \beta$-Unsaturated Esters with $\mathrm{CO} 2$ under Flow for $\beta$ Lactone Synthesis. ACS Catal. 2021, 11 (3), 1309-1315.

(48) Huang He; Ye Jian-Heng; Zhu Lei; Ran Chuan-Kun; Miao Meng; Wang Wei; Chen Hanjiao; Zhou Wen-Jun; Lan Yu; Yu Bo; Yu Da-Gang. Visible-Light-Driven Anti-Markovnikov Hydrocarboxylation of Acrylates and Styrenes with CO2. CCS Chem. $O$ (0).

(49) Alkayal, A.; Tabas, V.; Montanaro, S.; Wright, I. A.; Malkov, A. V.; Buckley, B. R. Harnessing Applied Potential: Selective $\beta$-Hydrocarboxylation of Substituted Olefins. J. Am. Chem. Soc. 2020, 142 (4), 1780-1785.

(50) Sheta, A.; Alkayal, A.; Malkov, A.; Buckley, B. Selective Electrosynthetic Hydrocarboxylation of Alpha,Beta-Unsaturated Olefins with Carbon Dioxide. 2021. 10.26434/chemrxiv.14381087.v2 (51) Hori, Y. Electrocarboxylation of Styrene through Homogeneous Redox Catalysis. J. Electroanal. Chem. Interfacial Electrochem. 1984, 177 (1-2), 303-309. https://doi.org/10.1016/00220728(84)80232-6.

(52) Hori, Y. Electrochemical CO2 Reduction on Metal Electrodes. In Modern Aspects of Electrochemistry; Vayenas, C. G., White, R. E., Gamboa-Aldeco, M. E., Eds.; Modern Aspects of Electrochemistry; Springer: New York, NY, 2008; pp 89-189.

(53) This strategy is analogous to established atom transfer radical addition reactions (ATRA) for a recent review of photocatalytic ATRA, see: Williams, T. M.; Stephenson, C. R. J. Atom Transfer Radical Addition Using Photoredox Catalysis. In Visible Light Photocatalysis in Organic Chemistry; John Wiley \& Sons, Ltd, 2018; pp 73-92.

(54) Chmiel, A.; Williams, O. P.; Chernowsky, C.; Yeung, C.; Wickens, Z. Non-Innocent Radical Ion Intermediates in Photoredox Catalysis: Parallel Reduction Modes Enable Coupling of Diverse Aryl Chlorides. J. Am. Chem. Soc. $\mathbf{2 0 2 1}$ Just accepted https://doi.org/10.1021/jacs.1c05988

(55) Wang, H.; Gao, Y.; Zhou, C.; Li, G. Visible-Light-Driven Reductive Carboarylation of Styrenes with $\mathrm{CO} 2$ and Aryl Halides. J. Am. Chem. Soc. 2020, 142 (18), 8122-8129.

(56) Hendy, C. M.; Smith, G. C.; Xu, Z.; Lian, T.; Jui, N. T. Radical Chain Reduction via Carbon Dioxide Radical Anion ( $\mathrm{CO}_{2}{ }^{--}$). J. Am. Chem. Soc. 2021, 143 (24), 8987-8992.

(57) For selected reviews of visible-light photoredox catalysis, see: $58-64$.

(58) Prier, C. K.; Rankic, D. A.; MacMillan, D. W. C. Visible Light Photoredox Catalysis with Transition Metal Complexes: Applications in Organic Synthesis. Chem. Rev. 2013, 113 (7), 5322-5363.

(59) Shaw, M. H.; Twilton, J.; MacMillan, D. W. C. Photoredox Catalysis in Organic Chemistry. J. Org. Chem. 2016, 81 (16), 68986926.

(60) Romero, N. A.; Nicewicz, D. A. Organic Photoredox Catalysis. Chem. Rev. 2016, 116 (17), 10075-10166.

(61) Skubi, K. L.; Blum, T. R.; Yoon, T. P. Dual Catalysis Strategies in Photochemical Synthesis. Chem. Rev. 2016, 116 (17), 1003510074 .

(62) DiRocco, D. A.; Dykstra, K.; Krska, S.; Vachal, P.; Conway, D. V.; Tudge, M. Late-Stage Functionalization of Biologically Active Heterocycles Through Photoredox Catalysis. Angew. Chem. Int. Ed. 2014, 53 (19), 4802-4806.

(63) Skubi, K. L.; Blum, T. R.; Yoon, T. P. Dual Catalysis Strategies in Photochemical Synthesis. Chem. Rev. 2016, 116 (17), 1003510074 .

(64) Narayanam, J. M. R.; Stephenson, C. R. J. Visible Light Photoredox Catalysis: Applications in Organic Synthesis. Chem. Soc. Rev. 2010, 40 (1), 102-113.

(65) For a special issue on photoredox catalysis in organic chemistry, see: Arora, A.; Weaver, J. D. Photocatalytic Generation of 2-Azolyl Radicals: Intermediates for the Azolylation of Arenes and 
Heteroarenes via C-H Functionalization. Org. Lett. 2016, 18 (16) 3996-3999. https://doi.org/10.1021/acs.orglett.6b01718.

(66) As part of a mechanistic study regarding their recently developed alkene arylcarboxylation reaction, $\mathrm{Li}$ and coworkers observed a single example of photoredox-catalyzed addition of formate across a 1,1-diphenylethylene radical trap, albeit in low yield (24\%). For details, see: Ref 55. Additionally, as we were preparing this manuscript Jui and coworkers reported an additional three examples of formate-based hydrocarboxylation of electron-deficient alkenes. For detail, see: Ref 56.

(67) Chernowsky, C.; Chmiel, A.; Wickens, Z. Electrochemical Activation of Diverse Conventional Photoredox Catalysts Induces New Potent Photoreductant Activity. Angew. Chem. Int. Ed. 2021 Just accepted. https://doi.org/10.1002/anie.202107169.

(68) Furthermore, when the reaction is shielded from light conversion of styrene is also not obsereved.

(69) Dénès, F.; Pichowicz, M.; Povie, G.; Renaud, P. Thiyl Radicals in Organic Synthesis. Chem. Rev. 2014, 114 (5), 2587-2693.

(70) Bordwell, F. G.; Zhang, X.-M.; Satish, A. V.; Cheng, J.-P. Assessment of the Importance of Changes in Ground-State Energies on the Bond Dissociation Enthalpies of the O-H Bonds in Phenols and the S-H Bonds in Thiophenols. J. Am. Chem. Soc. 1994, 116 (15), 6605-6610.

(71) For a review on polarity effects in hydrogen atom transfer reactions, see: Roberts, B. P. Polarity-Reversal Catalysis of Hydrogen-Atom Abstraction Reactions: Concepts and Applications in Organic Chemistry. Chem. Soc. Rev. 1999, 28 (1), 25-35.

(72) $\mathrm{KCHO}_{2}$ is $0.01 \mathrm{USD} / \mathrm{mmol}$ based on https://www.sigmaaldrich.com/US/en accessed July 19th, 2021.

(73) Under the standard $2 \mathrm{~h}$ reaction time, Ir-1 converts $19 \%$ styrene starting material. See SI for details.

(74) We noted that free carboxylic acids resulted in significant slower reaction times under standard conditions, presumably due to the change in $\mathrm{pH}$ of the system. To compensate, we added an excess of more soluble cesium formate.

(75) We suspect that this chemoselectivity is observed because addition of $\mathrm{CO} 2 \bullet-$ is rapid and sequesters active reductant but other possibilities cannot be ruled out.

(76) Harper, K. C.; Moschetta, E. G.; Bordawekar, S. V.; Wittenberger, S. J. A Laser Driven Flow Chemistry Platform for Scaling Photochemical Reactions with Visible Light. ACS Cent. Sci. 2019, 5 (1), 109-115.

(77) Roberts, D. Custom Carbon-14 Radiolabelling - Investing to Meet New Challenges. Drug Discovery World 2009, Winter, 53, (https://www.ddw-online.com/chemistry/p146740-custom-carbon-14-radiolabelling:-investing-to-meet-new-challenges.html, accessed July 19, 2021).

(78) For select recent examples of isotope exchange in the synthesis of carboxylic acids, see Ref 79-86.
(79) Destro, G.; Horkka, K.; Loreau, O.; Buisson, D.-A.; Kingston, L.; Vecchio, A. D.; Schou, M.; Elmore, C. S.; Taran, F.; Cantat, T.; Audisio, D. Transition-Metal-Free Carbon Isotope Exchange of Phenyl Acetic Acids. Angew. Chem. Int. Ed. 2020, 59 (32), 13490-13495.

(80) Gauthier, D. R.; Rivera, N. R.; Yang, H.; Schultz, D. M.; Shultz, C. S. Palladium-Catalyzed Carbon Isotope Exchange on Aliphatic and Benzoic Acid Chlorides. J. Am. Chem. Soc. 2018, 140 (46), 15596-15600.

(81) Babin, V.; Talbot, A.; Labiche, A.; Destro, G.; Del Vecchio, A.; Elmore, C. S.; Taran, F.; Sallustrau, A.; Audisio, D. Photochemical Strategy for Carbon Isotope Exchange with C02. ACS Catal. 2021, 11 (5), 2968-2976.

(82) Kong, D.; Munch, M.; Qiqige, Q.; Cooze, C. J. C.; Rotstein, B. H.; Lundgren, R. J. Fast Carbon Isotope Exchange of Carboxylic Acids Enabled by Organic Photoredox Catalysis. J. Am. Chem. Soc. 2021, 143 (5), 2200-2206.

(83) Destro, G.; Loreau, O.; Marcon, E.; Taran, F.; Cantat, T.; Audisio, D. Dynamic Carbon Isotope Exchange of Pharmaceuticals with Labeled C02. J. Am. Chem. Soc. 2019, 141 (2), 780-784.

(84) Kong, D.; Moon, P. J.; Lui, E. K. J.; Bsharat, O.; Lundgren, R. J. Direct Reversible Decarboxylation from Stable Organic Acids in Dimethylformamide Solution. Science 2020, 369 (6503), 557-561.

(85) Kingston, C.; Wallace, M. A.; Allentoff, A. J.; deGruyter, J. N.; Chen, J. S.; Gong, S. X.; Bonacorsi, S.; Baran, P. S. Direct Carbon Isotope Exchange through Decarboxylative Carboxylation. J. Am. Chem. Soc. 2019, 141 (2), 774-779.

(86) Tortajada, A.; Duan, Y.; Sahoo, B.; Cong, F.; Toupalas, G.; Sallustrau, A.; Loreau, O.; Audisio, D.; Martin, R. Catalytic Decarboxylation/Carboxylation Platform for Accessing Isotopically Labeled Carboxylic Acids. ACS Catal. 2019, 9 (7), 5897-5901.

(87) This reduction would be thermodynamically favorable from reduced 4DPAIPN. $E_{1 / 2}(\mathrm{PC} / \mathrm{PC} \bullet-)=-1.6 \mathrm{~V}$ vs SCE.

(88) The pKa of benzylic C-H bonds in DMSO is 43 and the $\mathrm{pKa}$ of DMSO itself is 35 . Water in DMSO has a pKa of 32.

(89) An ETPT mechanism that selectively engages $\mathrm{H} 2 \mathrm{O}$ but not DMSO cannot be excluded at this time.

(90) Cismesia, M. A.; Yoon, T. P. Characterizing Chain Processes in Visible Light Photoredox Catalysis. Chem. Sci. 2015, 6 (10), 5426-5434.

(91) Jacobsen, E.; Roberts, J. L.; Sawyer, D. T. Electrochemical Oxidation of Formate in Dimethylsulfoxide at Gold and Platinum Electrodes. J. Electroanal. Chem. Interfacial Electrochem. 1968, 16 (3), 351-360.

(92) An ${ }^{1}$ H NMR solubility assay indicated that DMSO saturated in potassium formate would result in approximately equimolar concentrations of $\mathbf{T} \mathbf{1}$ and potassium formate under standard reaction conditions. See SI for details. 


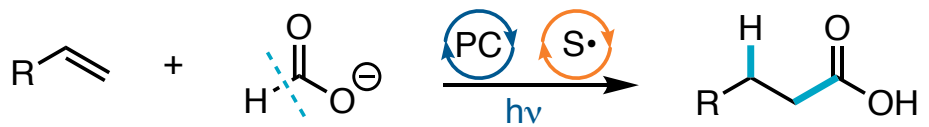
mild conditions - operationally simple " scaleable in batch 